\section{Louis Casteilla}

Béatrice Cousin

Nathalie Viguerie-

Bascands

Dominique Larrouy

Luc Pénicaud

\title{
Hétérogénéité et plasticité cellulaires des tissus adipeux
}

On distingue deux types de tissus adipeux : le tissu adipeux blanc et le tissu adipeux brun (TAB). Le premier constitue la plus grande réserve d'énergie de l'organisme, le second est un tissu spécialisé dans la dissipation d'énergie sous forme de chaleur. Ces tissus jouent un rôle capital dans la régulation de la balance énergétique de l'organisme, leur importance relative pouvant varier suivant les espèces. Comprendre le développement du TAB et les relations entre adipocytes bruns et adipocytes blancs est indispensable pour bien appréhender le développement de la masse adipeuse totale : une conversion tissulaire apparente a pu être observée, aussi bien au cours du développement que dans certaines conditions physiologiques ou pathologiques. Le système nerveux sympathique apparaît jouer un rôle majeur dans ces phénomènes de développement et de plasticité tissulaire et/ou cellulaire.

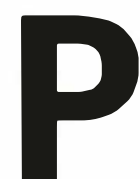

endant longtemps, on a considéré qu'il existait, chez les mammifères, deux types de tissus adipeux: les tissus adipeux blanc et brun contenant respectivement et exclusivement des adipocytes blancs et bruns.

\section{Tissus adipeux et adipocytes}

\section{ADRESSES}

L. Casteilla: professeur des universités. $\mathrm{N}$. Viguerie-Bascands : chargée de recherche $a$ l'INSERM. D. Larrouy : maitre de conférences des universités. Jeune équipe DRED, Biolodes universites. Jeune equipe DRED, Biolo-
gie des tissus adipeux, Bâtiment $\mathrm{Ll}, \mathrm{CHU}$ gie des tissus adipeux, Bâtiment L1, CHU
Rangueil, 1, avenue J.-Poulhès, 31054 Toulouse, France. B. Cousin : boursière $M R T$ L. Pénicaud : directeur de recherche au CNRS. URA 307, Université Paris-VII, 2, place Jussieu, 75251 Paris Cedex 05, France.
Le tissu adipeux brun (TAB) a été tout d'abord mis en évidence chez les animaux hibernants. Lorsqu'il est présent, il est impliqué dans la thermogenèse sans frisson et celle induite par l'alimentation [1]. Ce tissu est généralement localisé au contact immédiat des principaux vaisseaux sanguins, ce qui facilite la diffusion efficace à tout l'organisme de la cha- leur qu'il produit [2]. Ce tissu est richement vascularisé et innervé : on considère, en effet, que chaque adipocyte est au contact d'une terminaison nerveuse sympathique. Le système sympathique agit sur les tissus adipeux, et principalement le TAB, via la noradrénaline et des récepteurs $\beta$-adrénergiques. Un récepteur $\beta$-adrénergique relativement spécifique de l'adipocyte, le récepteur $\beta 3$ adrénergique a été caractérisé pharmacologiquement puis son gène cloné dans différentes espèces [3].

Les principales caractéristiques des deux types d'adipocytes sont rappelées dans le Tableau I. Ces deux types cellulaires sont capables de stocker des triglycérides, principalement sous l'effet de l'insuline et de les hydrolyser, essentiellement après stimulation par les catécholamines. 


\section{RÉFÉRENCES}

1. Himms-Hagen J. Brown adipose tissue thermogenesis : interdisciplinary studies. FASEB J $1990 ; 11$ : 2890-8.

2. Néchad M. Structure and development of brown adipose tissue. In : Trayhurn $P$, Nicholls DG, eds. Brown Adipose Tissue Londres : Edward Arnold, 1986:1-30.

3. Emorine L, Strosberg D. Structure et fonction du récepteur $\beta 3$-adrénergique. médecine/sciences $1993 ; 9 ; 1228-5$.

4. Ricquier D. Thermogénèse et obésité mécanismes moléculaires. médecine/sciences $1985 ; 1: 147-53$.

5. Ricquier D, Casteilla L, Bouillaud F. Molecular studies on the uncoupling protein. FASEB J 1991 ; 5 : 2237-42.

6. Silva J, Larsen PR. Adrenergic activation of triiodothyronine production in brown adipose tissue. Nature $1983 ; 305: 712-3$.

7. Young P, Arch JRS, Ashwell M. Brown adipose tissue in the parametrial fat pad of the mouse. FEBS Lett $1984 ; 167: 10-4$.

8. Cousin B, Cinti S, Morroni M, Raimbault S, Ricquier D Pénicaud L Casteilla L. Occurence of brown adipocytes in rat white adipose tissue : Molecular and morphological characterization. J Cell Science 1992; 103 : 931-42.

9. Gemmell RT, Bell AW, Alexander G. Morphology of adipose cells in lambs at birth and during subsequent transition of brown to white adipose tissue in cold and in warm conditions. Am J Anat $1972 ; 133$ : 14364.

10. Casteilla L, Champigny $\mathrm{O}$, Bouillaud $\mathrm{F}$, Robelin J, Ricquier D. Sequential changes in the expression of mitochondrial protein mRNA during the developement of BAT in bovine and ovine species : sudden occurence of Uncoupling protein mRNA during embryogenesis and its disappearance after birth. Biochem J $1989 ; 257: 665-71$.

11. Soppela P, Nieminen M, Saarela S, Keith JS, Morrison JN, MacFarlane F, Trayhurn P. Brown fat-specific mitochondrial uncoupling protein in adipose tissues of newborn reindeer. Am J Physiol 1991 ; 260 . R1229-34.

12. Vatnick I, Tysbir RS, Welch JG, Hooper AP. Regression of brown adipose tissue mitochondrial function and structure in neonatal goats. Am J Physiol 1987 ; 252 : E391-5.

13. Lean MEJ, James WPT. Brown adipose tissue in man. In Trayhurn P, Nicholls DG eds. Brown adipose tissue. Londres : Edward Arnold, $1986: 339-65$.

14. Champigny O, Blondel O, Ricquier D Mayers RM, Briscoe MG, Holloway BR. B3adrenoreceptor stimulation restores message and expression of brown fat mitochondrial uncoupling protein (UCP) in adult dogs. Proc Nat Acad Sci USA 1991

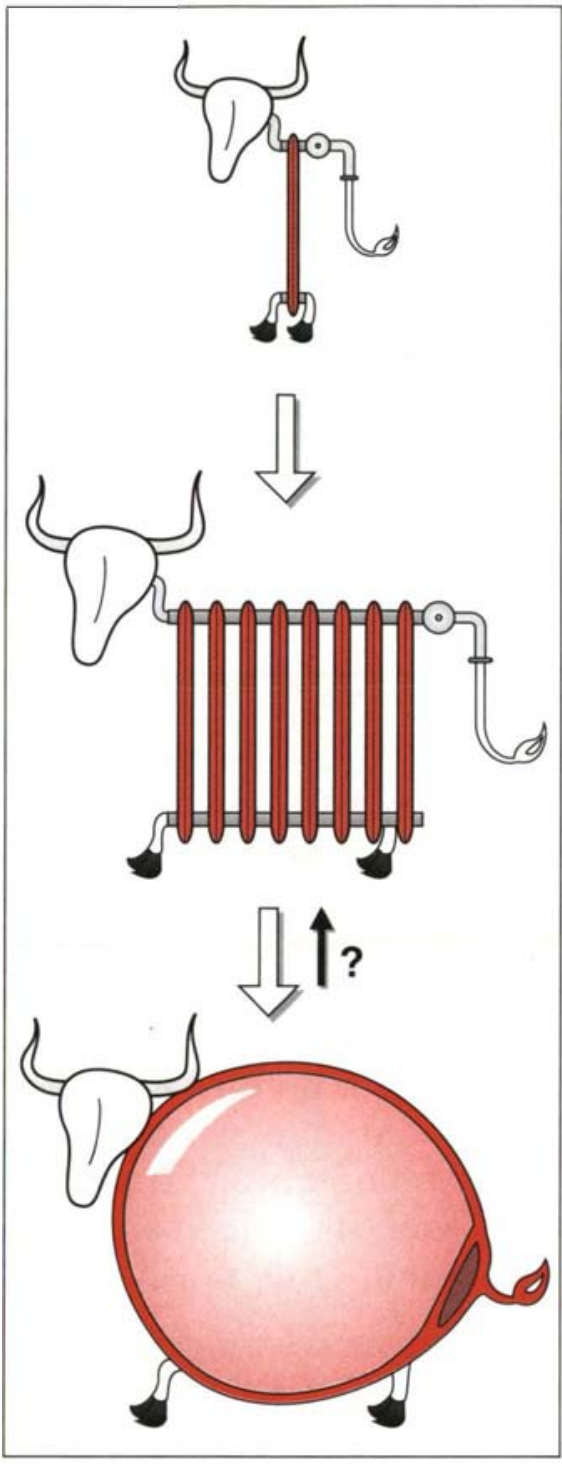

Figure 1. Ontogenèse du TAB chez les ruminants. Ce dessin résume le développement in vivo du TAB et sa transformation post-natale chez les ruminants. Le TAB qui est un organe thermogénique est représenté ici par un radiateur. II acquiert progressivement toutes ses caractéristiques in utero. A la naissance, la majorité des dépôts adipeux est constituée de dépôts adipeux bruns dont les adipocytes expriment la protéine découplante ou UCP. Ces tissus représentent de $2 \%$ à $4 \%$ du poids corporel du nouveau-né. Rapidement après la naissance, les tissus perdent leurs caractéristiques et se transforment en tissu semblable à du tissu adipeux blanc dont les adipocytes sont uniloculaires et ont perdu leur fonction thermogénique. La possibilité de revenir à un phénotype brun n'a jamais été décrite chez ces espèces mais est réalisée dans certaines situations physiopathologiques ou après un traitement avec un agoniste $\beta 3$-adrénergique chez le chien [13].

Des caractéristiques morphologiques, biochimiques et fonctionnelles permettent cependant de différencier l'adipocyte blanc de l'adipocyte brun. L'adipocyte blanc mûr est uniloculaire, contient peu de cytoplasme avec peu de mitochondries. L'adipocyte brun est multiloculaire et très riche en mitochondries très actives. Par découplage de sa respiration mitochondriale, il a la capacité de convertir en chaleur l'énergie libérée par l'oxydation des acides gras [4]. La protéine mitochondriale (UCP pour uncoupling protein) responsable de ce phénomène a été détectée, à l'heure actuelle, dans ce seul type cellulaire [5]. Il existe une corrélation étroite entre le contenu en cette protéine, dont l'expression est contrôlée au niveau transcriptionnel par les catécholamines, et le potentiel thermogénique du tissu. L'adipocyte brun possède une enzyme, la T4-5'déiodase, qui est capable de convertir la T4 circulante en triiodothyronine. L'activité de cette enzyme est indispensable pour la transcription maximale du gène de l'UCP et la réponse au froid des adipocytes bruns [6].

\section{Hétérogénéité cellulaire des dépôts adipeux}

Pendant longtemps, il a semblé que les deux tissus étaient totalement dissociés et homogènes et que 
Tableau I

PRINCIPALES CARACTÉRISTIQUES DES DEUX TYPES D'ADIPOCYTES

\begin{tabular}{|c|c|c|}
\hline Caractéristiques & Tissu adipeux brun & Tissu adipeux blanc \\
\hline Tissulaires & $\begin{array}{l}\text { Localisé principalement autour } \\
\text { des gros vaisseaux } \\
\text { Vascularisation (+++) } \\
\text { Innervation }(+++) \\
\text { Cellules endothéliales }(+++)\end{array}$ & $\begin{array}{l}\text { Localisation plus diffuse } \\
\text { Vascularisation }(++) \\
\text { Innervation }(+) \\
\text { Cellules endothéliales (+) }\end{array}$ \\
\hline $\begin{array}{l}\text { Morphologie cellulaire } \\
\text { des adipocytes }\end{array}$ & $\begin{array}{l}\text { Multiloculaire } \\
\text { Richesse en mitochondries } \\
\text { Grains de glycogène }\end{array}$ & $\begin{array}{l}\text { Uniloculaire } \\
\text { Peu de cytoplasme }\end{array}$ \\
\hline Fonctionnelles & $\begin{array}{l}\text { Stimulation par la noradrénaline: } \\
\text { - Utilisation d'acides gras et libération } \\
\text { d'énergie sous forme de chaleur } \\
\text { - Stimulation de la lipoprotéine lipase } \\
\text { Production de triiodothyronine } \\
\text { (nouveau-né au froid) } \\
\text { Sensibilité à l'insuline (++++) }\end{array}$ & $\begin{array}{l}\text { Stimulation par la noradrénaline: } \\
\text { - Libération d'énergie sous forme } \\
\text { d'acides gras } \\
\text { - Inhibition de la lipoprotéine lipase } \\
\text { Sensibilité à l'insuline (++) }\end{array}$ \\
\hline $\begin{array}{l}\text { Biochimiques et moléculaires } \\
\text { spécifiques }\end{array}$ & $\begin{array}{l}\text { Protéine découplante ou UCP } \\
\text { T4-5' Déiodase très active }\end{array}$ & \\
\hline
\end{tabular}

l'ensemble des adipocytes bruns était localisé dans des dépôts adipeux bruns, distincts des dépôts de tissu adipeux blanc. Cette notion est à présent remise en question. La présence en faible quantité de protéine découplante avait été observée dans le tissu adipeux paramétrial de souris maintenues à $23{ }^{\circ} \mathrm{C}$ [7]. Depuis, la présence d'adipocytes bruns a pu être caractérisée en quantité non négligeable et variable au sein des dépôts adipeux blancs chez des rats maintenus à une température de $22{ }^{\circ} \mathrm{C}$ [8]. Ces résultats indiquent que les adipocytes bruns ne sont pas uniquement localisés dans les dépôts de TAB. Ces résultats peuvent être étendus aux primates. Dans une étude en cours, nous avons pu caractériser par immunocytochimie la présence d'adipocytes bruns, ou d'îlots d'adipocytes bruns, au sein de dépôts adipeux blancs (Viguerie-Bascands, Bousquet, Larrouy, Galitzky, Berlan, Casteilla, résultats non publiés). L'hypothèse selon laquelle la classification du tissu adipeux ne se limiterait pas à deux formes distinctes se voit donc confortée par ces observations. Pour un même animal, l'ensemble des tissus adipeux constituerait un gradient ou continuum de tissus au sein duquel la richesse en adipocytes bruns varierait dans une large proportion. A l'une des extrémités, le tissu adipeux brun "typique ", semblable au TAB interscapulaire chez le rat, au sein duquel la très grande majorité des adipocytes sont des adipocytes bruns ; à l'autre extrémité, le tissu adipeux blanc caractéristique tel que le tissu adipeux épididymaire qui est essentiellement constitué d'adipocytes blancs.

\section{Conversion et plasticité au niveau tissulaire}

Un certain nombre d'arguments sont en faveur d'une conversion phénotypique possible entre les tissus adipeux blancs et bruns, ce qui révèle leur caractère plastique. Il existe de nombreuses situations physiologiques ou pathologiques s'accompagnant d'une conversion apparente de ces tissus.

Le TAB se met en place essentiellement pendant la vie intra-utérine et est présent en quantité importante chez le nouveau-né. La masse du tissu adipeux blanc se développe principalement après la naissance. Chez les gros mammifères et en particulier chez les ruminants, on observe au cours du développement, une disparition progressive des dépôts adipeux ayant les caractéristiques histologiques et biochimiques du TAB. Ces tissus perdent ces spécificités pour se transformer en tissu adipeux semblable au tissu adipeux blanc (figure 1) [9-12]. Dans l'espèce humaine, le $\mathrm{TAB}$ persiste plus longtemps après la naissance mais cette transformation apparente du TAB en tissu adipeux blanc semble exister [13].

Ce processus semble irréversible, du moins chez les ruminants, puisque, même lorsque les jeunes animaux sont élevés à $4{ }^{\circ} \mathrm{C}$, il n'est possible que de le retarder [9]. Ce n'est pas le cas chez le chien. Dans cette espèce, un traitement pharmacologique avec un agoniste $\beta 3$-adrénergique entraîne, dans tous les dépôts étudiés, la réapparition en quantité importante d'adipocytes bruns mis en évidence par la détection des ARNm de l'UCP [14]. Différents travaux ont permis d'étendre ces observations aux rongeurs. Ainsi, l'exposition chronique au froid de souris ou de rats entraîne l'apparition en nombre très important de cellules multiloculaires enrichies en mitochondries et exprimant la protéine découplante dans des dépôts adipeux définis comme blancs $[7,8$, 15]. L'auteur de cette dernière 


\section{RÉFÉRENCES}

15. Loncar D. Convertible adipose tissue in mice. Cell Tissue Res 1991 ; 266 : 149-61.

16. Ricquier D, Mory G. Factors affecting brown adipose tissue in animals and man. Clin Endocrinol Metab 1984 ; 13 : 501-520.

17. Huttunen P, Hirvonen J, Kinnula V. The occurence of brown adipose tissue in $46: 339-45$.

18. Garruti G, Ricquier D. Analysis of uncoupling protein and its mRNA in adipose deposits of adult humans. Int J Obes $1992 ; 16: 383-90$.

19. Ashwel M. Is there a continuous spectrum of the adipose tissues in animals and man? In : Vague J, eds. Metabolic complications of human obesities. New York: Elsevier Science Publishers Bv. 1985 : 265-74.

20. Casteilla L, Forest C, Robelin J Ricquier D, Lombet A, Ailhaud $G$. Characterization of mitochondrial uncoupling protein in bovine fetus and newborn calf. Disappearance in lamb during aging. Am J Physiol 1987 ; 252 : E62736 .

21. Langin D, Portillo $M$, Dauzats $M$, Lafontan $M$. Drop in the atypical $\beta$ adrenergic response and modification of the $\beta / \alpha 2$-adrenoceptor balance in fat cells from aging rabbits. Endocrinology $1992 ; 130$ : $307-15$.

22. Casteilla L, Muzzin P, Révelli IP, Ricquier D, Giacobino JP. Expression of $\beta 1$ and 133 -adrenergic-receptor messages and adenylate cyclase $\beta$-adrenergic response in bovine perírenal adipose tissue during its transformation from brown into white fat. Biochem J 1994 ; 297 : 93-7.

23. Galitzky J, Reverte $M$, Portilla $M$, Carpéné $C$, Lafontan $M$, Berlan $M$ Coexistence of functional $\beta 1-, \beta 2-$ and $\beta 3$. adrenoceptors in dog fat cells and their differential activation by catcholamines. $A m$ JPhysiol 1993 ; 264 : E403-12.

24. Bukowiecki LJ, Collet AJ, Folléa N Guay G, Jahjah L. Brown adipose tissue hyperplasia : a fundamental mechanism of adaptation to cold and hyperphagia. $A m J$ Physiol $1982 ; 242$ : E353-9

25. Géloën A, Collet AJ, Guay G, Bukowiecki LJ. $\beta$-adrenergic stimulation of brown adipocyte proliferation. Am J Physiol 1988 ; 254 : C175-82.

26. Rehnmark S, Nechad M, Herron D, Cannon B, Nedergaard J. Alpha and betaadrenergic induction of the expression of the uncoupling protein thermogenin in brown adipocytes differentiated in culture. J Biol Chem 1990 ; 265 : 16464-71.

27. Casteilla L, Nouguès J, Reyne $Y$, Ricquier D. Differentiation of ovine brown preadipocytes in a chemically defined serum-free medium. Effect of glucocorticoids and age of donor. Eur Biochem 1991; 198:195-9. étude conclut à une conversion apparente et massive de ces tissus en $\mathrm{TAB}$ et les qualifie de tissus adipeux convertibles [15]. La réapparition d'adipocytes bruns caractéristiques a également été observée chez l'homme. En effet, dans plusieurs situations pathologiques (phéochromocytome, hyperaldostéronémie) ou physiologiques extrêmes (travailleurs exposés au froid), la présence d'UCP et d'adipocytes bruns a été détectée dans des régions où le tissu adipeux est normalement de type blanc $[13,16-18]$.

Si l'on considère l'ensemble des espèces, il est possible d'élargir la notion du continuum cité précédemment suivant le degré de convertibilité des dépôts $[7,8,15,19]$. Celui-ci irait du TAB de l'hibernant au tissu adipeux blanc caractéristique des mammifères de grande taille dont le phénotype ne peut être modifié, en passant par les tissus convertibles. Une plasticité relative existerait à l'intérieur de ce spectre suivant les conditions physiologiques, pathologiques ou expérimentales.

\section{L'hypothèse de \\ l'adipocyte brun masqué}

La transformation observée au niveau tissulaire peut-elle être liée à l'interconversion, à un niveau cellulaire, d'adipocytes bruns en adipocytes blancs et inversement? On ne sait pas à l'heure actuelle si cette conversion apparente au niveau tissulaire correspond à la transformation de cellules préexistantes ou résulte de la prolifération et de la différenciation de nouveaux précurseurs. Trois hypothèses non exclusives permettent d'expliquer ces modifications phénotypiques (figure 2). Dans le premier cas, il existerait une seule population adipocytaire qui possèderait le phénotype blanc ou brun en fonction du stade de développement ou des conditions environnementales (hypothèse 1). Dans le deuxième cas, il existerait deux populations différentes d'adipocytes, qui n'auraient aucune possibilité d'interconversion; dans ce cas, l'apparition de nouvelles cellules brunes au sein des dépôts blancs serait uniquement due au recrutement de précurseurs adipocytaires bruns (hypothèse 2). Enfin, dans la troisième hypothèse, il existerait trois populations d'adipocytes, des adipocytes blancs et bruns dont les caractéristiques seraient invariables, et des adipocytes dont le phénotype pourrait se transformer (hypothèse 3) ; dans ces conditions, ces adipocytes seraient des adipocytes bruns "dormants " ou * masqués " dont le phénotype caractéristique pourrait réapparaître dans certaines conditions physiologiques (adaptation au froid), pathologiques (phéochromocytome, hyperaldostéronémie) ou pharmacologiques (traitement par un agoniste $\beta$-adrénergique).

La première hypothèse paraît peu probable. En effet, in vivo, même dans des conditions extrêmes comme l'exposition prolongée au froid, les deux phénotypes sont présents. Il existe quelques arguments indirects en faveur de la dernière hypothèse. Chez les ruminants, la transformation du TAB après la naissance, en tissu adipeux blanc intervient alors que le nombre d'adipocytes différenciés ne varie pas [9, 20]. Au cours de l'analyse histologique par microscopie électronique de cette transformation, toutes les "figures" intermédiaires entre un adipocyte brun caractéristique et un adipocyte blanc ont pu être identifiées [9]. De même, chez les rongeurs, des analyses histologiques et immunocytochimiques montrent la présence de cellules exprimant l'UCP et ayant une morphologie intermédiaire entre adipocyte blanc et adipocyte brun [8, 15]. Ces arguments sont clairement en faveur de l'interconvertibilité de certains adipocytes bruns en adipocytes qui ressemblent à des adipocytes blancs. Ces cellules sont-elles strictement identiques à de vrais adipocytes bruns? A l'heure actuelle, aucun critère biochimique ou moléculaire ne permet de les différencier, mais cette question mérite d'être débattue.

On doit souligner ici, que l'importance des effets biologiques liés à l'utilisation d'agents agonistes B3adrénergiques est parallèle au continuum entre les tissus adipeux brun et blanc décrit précédemment [21]. $\mathrm{Si}$ le récepteur $\beta 3$-adrénergique est le récepteur majoritairement exprimé pendant la période foetale et périnatale chez le bovin, son expres- 


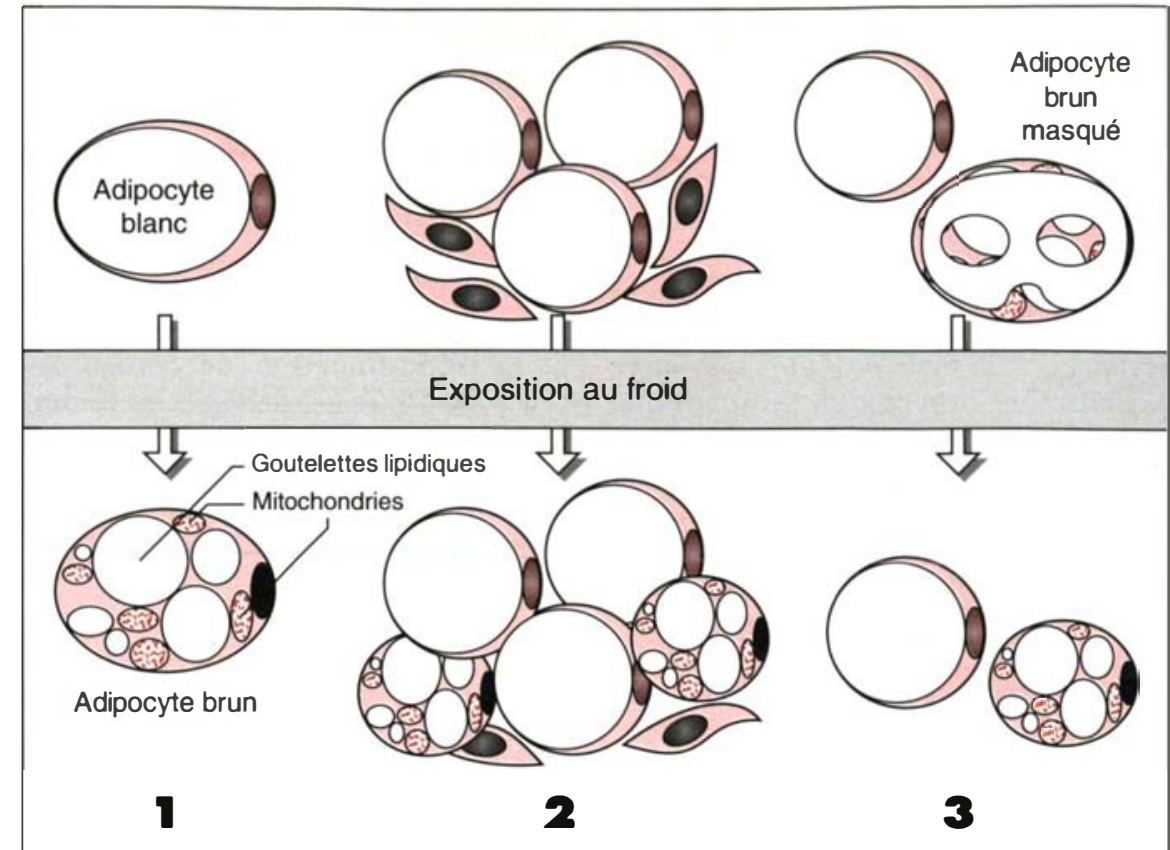

Figure 2. Hypothèses à l'échelle cellulaire permettant d'expliquer l'augmentation du nombre d'adipocytes bruns dans les dépôts blancs, conduisant à une conversion du phénotype tissulaire. A la suite de l'adaptation à $4{ }^{\circ} \mathrm{C}$ de rongeurs, le nombre d'adipocytes bruns augmente dans les tissus adipeux blancs. Trois hypothèses permettraient d'expliquer cette augmentation lde gauche à droite sur le dessin). Hypothèse 1 : la conversion totale entre phénotypes cellulaires est possible. Hypothèse 2 : il n'existe pas de conversion ; dans ces conditions, l'apparition de nouvelles cellules adipeuses brunes est due exclusivement à la différenciation d'adipocytes bruns à partir des précurseurs. Hypothèse 3: certaines cellules semblables à des adipocytes blancs sont en fait des adipocytes bruns dont le phénotype caractéristique est masqué : les gouttelettes lipidiques ont tendance à fusionner et les mitochondries à disparaître; le phénotype spécifique de l'adipocyte brun réapparaîtrait à la suite de l'exposition des animaux à $4^{\circ} \mathrm{C}$. Bien évidemment aucune de ces hypothèses n'est exclusive d'une autre. Sur cette figure, les adipocytes blancs et bruns sont schématisés par leurs caractéristiques histologiques et des noyaux de couleurs différentes : à savoir, l'aspect uniloculaire, peu de cytoplasme et un noyau bistre pour l'adipocyte blanc et l'aspect multiloculaire, de nombreuses mitochondries et un noyau noir pour l'adipocyte brun. Les précurseurs adipocytaires sont schématisés sous forme de fibroblastes.

sion diminue de manière considérable au cours du développement jusqu'à devenir pratiquement indécelable [22]. Chez le chien, le récepteur $\beta 3$-adrénergique est présent dans les dépôts adipeux blancs [23]. Or, pour cette même espèce, un traitement pharmacologique avec un agoniste $\beta 3$-adrénergique entraîne la réapparition de l'UCP dans tous ces dépôts adipeux qui étaient négatifs chez les animaux témoins [14]. Ces $\mathrm{m} / \mathrm{s} n^{\circ} 11$, vol. 10, novembre 94
$[24,25]$. Dans le cas des tissus " convertibles", si la " conversion" cellulaire peut expliquer en partie, et suivant les dépôts, l'apparition rapide de nouveaux adipocytes bruns, un recrutement de cellules précurseurs aptes à se différencier n'est pas exclu.

Bien qu'il soit couramment admis que les adipocytes blancs et bruns aient pour origine le mésoderme, on ne sait pas à l'heure actuelle si les deux types d'adipocytes dérivent d'un précurseur unique ou s'il existe deux types de précurseur. Cependant, des cellules issues de la fraction du stroma vasculaire de tissus adipeux brun et blanc mises en culture dans des conditions identiques de milieu défini, se différencient respectivement en adipocytes bruns et blancs [26-28]. La capacité de ces précurseurs de se différencier pourrait évoluer au cours du développement. En effet, les préadipocytes issus du tissu adipeux périrénal d'un agneau nouveau-né peuvent se différencier en adipocytes bruns mais, un mois plus tard, des préadipocytes issus du même dépôt perdent la capacité de se différencier en adipocyte brun [27]. Quoi qu'il en soit, ces résultats permettent de proposer qu'il existe au moins un stade, proche de la différenciation terminale, auquel on peut distinguer les précurseurs aptes à se différencier en adipocytes bruns, des précurseurs aptes à se différencier en adipocytes blancs.

\section{Système nerveux sympathique et plasticité tissulaire}

Les conditions physiologiques et pathologiques associées aux modifications phénotypiques des tissus adipeux sont caractérisées pour la plupart par une modification de l'activité sympathique.

L'effet positif de l'innervation sympathique sur le maintien et le développement du $\mathrm{TAB}$ a été très étudié [29]. La majeure partie des effets observés (stimulation de la prolifération et de la différenciation) peut être reproduite par l'administration de noradrénaline à l'animal [25]. Depuis ces études in vivo, les études in vitro ont confirmé ces résultats. La noradrénaline ou un analogue $\beta$ - 


\section{RÉFÉRENCES}

28. Ailhaud G. Some new aspects on adipose tissue development. Diab Metab Rev $1992 ; 8: 3-7$.

29. Girardier L, Seydoux J. Neural control of brown adipose tissue. In Trayhurn $P$ Nicholls DG eds. Brown adipose tissue. London : Edward Arnold, 1986 : 122-51.

30. Bronnikov G, Houstek J, Nedergaard J. $\beta$-adrenergic, cAMP-mediated stimulation of proliferation of brown fat cells in primary culture. Mediation via $\beta 1$ but not via $\beta 3$ adrenoceptors. J Biol Chem $1992 ; 267$ : 200613.

31. Lafontan $M$, Berlan $M$. Fat cell adrenergic receptors and the control of white and brown fat cell function. J Lipid Res 1993 ; 34 : 1057-91.

32. Ibrahimi A, Bertrand B, Bardon $S$, Amri E, Grimaldi P, Ailhaud $G$, Dani $C$. Cloning of $\alpha 2$ chain of type VI collagen and expression during mouse development. Biochem /1993; 289 : 141-7.

33. Cousin B, Casteilla L, Lafontan $M$, Ambid L, Langin D, Berthault MF, Pénicaud L. Local sympathetic denervation of white adipose tissue in rats induces preadipocyte proliferation without noticeable changes in metabolism. Endocrinology 1993 ; $133: 2255-62$.

34. Symonds ME, Bryant MJ, Clarke L, Darby CJ, Lomax MA. Effect of maternal cold exposure on brown adipose tissue and thermogenesis in the neonatal lamb. $J$ Physiol $1992 ; 455: 487-502$.

35. Klein AH, Reviczky A, Chou $P$, Padbury J, Fisher DA. Development of brown adipose tissue thermogenesis in the ovine fetus and newborn. Endocrinology $1983 ; 112: 1662-6$.

36. Douglas M. Could a lowered level of uncoupling protein in brown adipose tissue mitochondria play a role in SIDS aetiology. Med Hypothesis $1992 ; 37: 100-2$

37. Andrews DC, Symonds ME, Johnson P. The interaction of the upper airway and the thermometabolism on respiratory rhythm during N-REM sleep in developing lamb. $J$ Dev Physiol 1991 ; 16 : 38-44.

38. Symonds ME, Andrews DC, Johnson $P$. The control of thermoregulatory in the developing lamb during slow wave sleep. $J$ Dev Physiol 1989 : 289-98.

39. Jennings G, Elia M. Effect of E. Coli endotoxin on temperature oxygen consumption and brown adipose tissue consumption and brown adipose tissue thermogenesis in
$1987 ; 7: 517-23$.

40. Jepson MM, Millward DJ, Rothwell NJ, Stock MJ. Involvement of sympathetic nervous system and brown fat in endotoxininduced fever in rats. Am J Physiol 1988 ; induced fever
$255:$ E617-20.

41. Lowell BB, S-Susulic V, Hamann A, Lawitts JA, Himms-Hagen J, Boyer BB, Kozak L, Flier J. Development of obesity in transgenic mice after genetic ablation of brown adipose tissue. Nature $1993 ; 366$ : adrénergique, l'isoprotérénol, stimule la prolifération des cellules précurseurs des adipocytes bruns et facilite leur différenciation [30]. Leurs effets sur le contrôle de l'expression du gène de l'UCP sont par ailleurs bien décrits [5].

Alors que le rôle des catécholamines circulantes sur le métabolisme adipocytaire a été bien étudié, l'influence in vivo de l'innervation sympathique sur la cellularité du tissu adipeux blanc n'est que peu connue [31]. Afin d'étudier les effets de l'innervation sympathique sur le nombre et la taille des cellules adipeuses constituant ce tissu, il est possible de dénerver chirurgicalement et unilatéralement un dépôt bien identifié anatomiquement: le dépôt rétropéritonéal. L'effet majeur de cette intervention est une augmentation importante de la quantité d'ADN dans ce tissu qui traduit une hyperplasie massive. L'utilisation d'un marqueur exprimé précocement et de manière transitoire a cours de la différenciation terminale adipocytaire - A2COL6 codant pour la chaîne $\alpha 2$ du collagène de type VI [32] -, permet de montrer que cette hyperplasie correspond à un recrutement de préadipocytes qui quelques jours plus tard auront toutes les caractéristiques d'adipocytes blancs [33]. Parallèlement à ce recrutement de cellules précurseurs, les adipocytes déjà différenciés expriment des marqueurs tardifs de cette même différenciation tel que le récepteur adrénergique de type $\alpha 2$. L'innervation sympathique aurait, in vivo, un rôle inhibiteur sur le recrutement de précurseurs des cellules adipocytaires blanches et sur la différenciation terminale de ces cellules.

Les conclusions de ces deux derniers paragraphes sont résumées dans la figure 3. L'ensemble de ces résultats obtenus in vivo comme in vitro montre qu'il existe des voies différentes selon les programmes de différenciation et que le phénotype adipocytaire blanc n'est pas uniquement un stade très mûr de l'adipocyte brun [28]. L'innervation sympathique semble avoir un effet opposé sur la cellularité des deux types de tissus adipeux et permettre ainsi leur réorganisation en fonction des besoins de l'individu. Dans le tissu adipeux blanc, l'activité sympathique retarde la prolifération et la différenciation des précurseurs, alors que dans le $\mathrm{TAB}$, le phénomène inverse est observé. Dans le cas du TAB, la prolifération de précurseurs augmente le potentiel thermogénique du tissu et sa capacité de dissiper l'énergie, alors que dans le cas du tissu adipeux blanc, la prolifération et la différenciation des préadipocytes facilitent le stockage de l'énergie. A l'heure actuelle, aucun résultat in vitro ne permet de proposer que certains préadipocytes puissent être réorientés. In vivo, aucune donnée ne permet de conclure. Au cours du développement ou après dénervation, les adipocytes bruns peuvent perdre leurs caractéristiques spécifiques dont l'expression de l'UCP et ressembler à des adipocytes blancs. Ces cellules sont-elles strictement identiques à de vrais adipocytes blancs? L'absence de marqueurs ne permet pas de répondre à cette question mais, il semble clair que tous les adipocytes blancs n'ont pas la capacité de se convertir en adipocyte brun même après une exposition prolongée au froid [33]

\section{Tissu adipeux et physiopathologie}

Les capacités de prolifération différentielle et donc de réarrangement tissulaire peuvent avoir leur importance dans certaines problématiques médicales telles que la périnatalité et l'obésité.

\section{Tissus adipeux et périnatalité}

Chez les espèces de grande taille, dont l'homme, on peut considérer que le TAB occupe $1 \%$ à $2 \%$ du poids corporel chez le nouveau-né. Nous avons vu que la naissance représente une étape clé dans le développement du tissu adipeux et que la majorité des études concernant ce domaine a été effectuée chez les ruminants. Durant la gestation, le développement du tissu adipeux brun in utero, et donc les capacités thermogéniques ultérieures du nouveau-né, sont sensibles à la température extérieure à laquelle est exposée la mère [34]. La consommation d'oxygène de $T A B$ périrénal prélevé chez des fotus ovins est sti- 


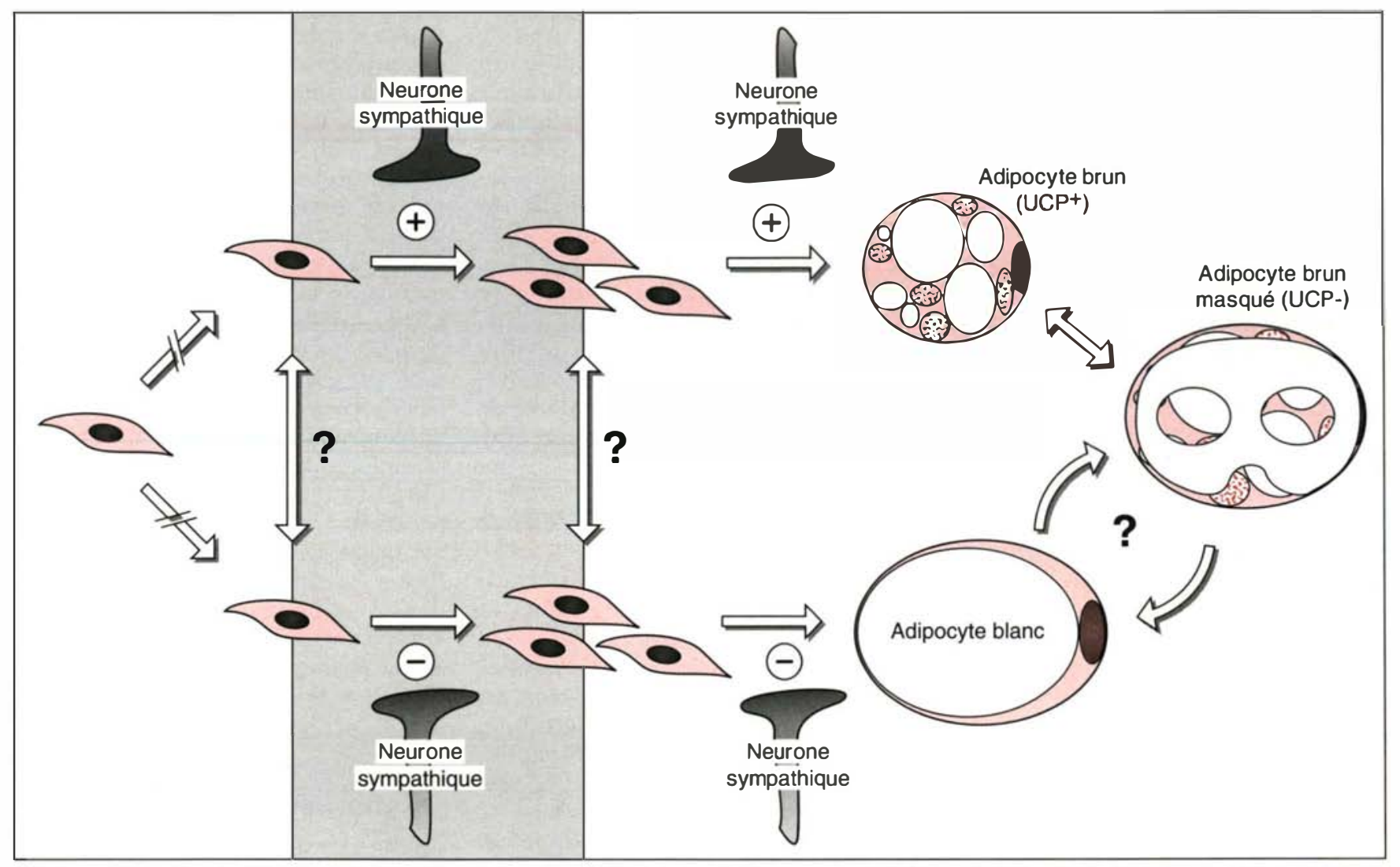

Différenciation des précurseurs à partir d'une cellule souche
Recrutement de préadipocytes
Acquisition du phénotype mûr

Figure 3. Recrutement de précurseurs et différenciation terminale des adipocytes blancs et bruns : influence de l'innervation sympathique. L'innervation sympathique est responsable du recrutement des préadipocytes bruns et facilite leur différenciation terminale in vivo (partie supérieure du dessin). A l'opposé, cette même innervation agirait comme un frein sur le recrutement des préadipocytes blancs et l'acquisition des caractéristiques du phénotype mûr (partie inférieure du dessin). De nombreux arguments indirects sont en faveur de la transformation d'adipocytes bruns qui expriment la protéine découplante (adipocyte brun UCP+) en cellules adipeuses semblables à des adipocytes blancs que l'on pourrait qualifier d'adipocytes bruns masqués qui n'expriment plus cette même protéine (adipocyte brun masqué UCP-). Ces cellules sont-elles équivalentes à de vrais adipocytes blancs bien qu'elles puissent retrouver leur phénotype initial dans différentes conditions? Quelles sont les relations entre les précurseurs ? Est-il possible, in vivo, de modifier leur programmation et de convertir un précurseur adipocytaire brun en précurseur adipocytaire blanc ? De nombreuses interrogations figurées par un point d'interrogation sur le dessin restent à élucider. Les principales étapes de la différenciation adipocytaires discernables in vivo et prises en considération dans le dessin sont identifiées dans les cadres inférieurs.

mulée par les catécholamines et les hormones thyroïdiennes [35] ce qui semble indiquer que le $\mathrm{TAB}$ est fonctionnel in utero.

Dans l'espèce humaine, la régulation de la température corporelle par une thermogenèse active n'est pas un problème crucial dans les sociétés occidentales mais a pu représenter un problème au cours de l'évolution. Quoi qu'il en soit, une hypo ou hyperactivation du TAB pourrait $\mathrm{m} / \mathrm{s} n^{\circ} 11$, vol. 10 , novembre 94 intervenir dans des affections périnatales non encore expliquées ainsi que chez des individus vivant dans des conditions extrêmes et/ou mal protégés [13,36].

L'implication directe et l'importance réelle d'un dysfonctionnement du TAB dans la mort subite du nourrisson est sujette à de nombreuses discussions et controverses mais mérite d'être explorée [36]. Il n'existe pas à l'heure actuelle d'arguments dé- monstratifs mais une relation entre le fonctionnement du TAB et le contrôle respiratoire peut être établie en période périnatale et cet accident serait associé à un dérèglement des centres respiratoires durant le sommeil. En effet, chez l'agneau nouveau-né, il n'est possible d'induire une respiration irrégulière que lorsque, simultanément, l'animal respire faiblement et est exposé à des températures aux- 
quelles le TAB n'est pas mis en jeu (températures proches de la limite supérieure de la thermoneutralité) [37]. Par ailleurs, sur douze agneaux utilisés lors d'une étude sur la thermorégulation, quatre animaux à la consommation d'oxygène basale significativement plus faible sont morts en cours d'expérimentation [38]. Cette dépense énergétique de base plus faible peut être associée à une capacité thermogénique plus faible du TAB [34]. Ainsi, un dysfonctionnement du TAB ou de son activation, associé à des températures ambiantes peu propices à sa stimulation, pourrait avoir des effets importants sur le contrôle de la respiration et la survie du nouveau-né. Enfin, plusieurs travaux démontrent que la production de chaleur par le $\mathrm{TAB}$ peut participer à la fièvre après une infection ou l'administration de lipopolysaccharides [39, 40]. Étant donné les capacités thermogéniques de ce tissu et sa masse à la naissance, il est fort probable qu'un dérèglement puisse avoir des conséquences néfastes pour l'organisme.

Tout cela reste, bien évidemment, à démontrer de manière définitive dans l'espèce humaine.

\section{Tissus adipeux et obésité}

L'obésité et ses implications pathologiques sont parmi les principaux dérèglements de l'organisme observés dans les sociétés occidentales. Le syndrome d'obésité est caractérisé principalement par un développement excessif du tissu adipeux blanc par rapport à la masse maigre de l'organisme.

Quel que soit le modèle d'obésité chez les rongeurs, génétique ou induite, l'hypertrophie de la masse adipeuse blanche est associée à une diminution de la masse et de l'activité thermogénique du TAB. Ce défaut de dissipation calorique participe à l'installation de l'obésité, en réduisant la dépense énergétique sous forme de chaleur. L'hypotrophie du tissu adipeux brun est associée à une modification du phénotype cellulaire: disparition apparente du phénotype brun et apparition du phénotype blanc. Ces modifications peuvent être mises en relation avec une diminution de l'activité sympa-
Afin de déterminer si une destruction, la plus sélective possible du $\mathrm{TAB}$, était suffisante pour entraîner un excédent pondéral, des lignées de souris transgéniques porteuses d'un gène chimérique correspondant à la fusion du promoteur du gène de l'UCP avec le gène codant pour une toxine intracellulaire ont été établies [41]. Ces animaux se développent normalement mais, après la naissance, il apparaît une obésité importante et un diabète. Cependant, pour que cette démonstration soit définitive, et bien que cette expérimentation soit élégante, il est indispensable d'être sûr que l'expression de la toxine n'a affecté que le TAB.

Un autre axe important est celui ouvert par les observations de Symonds et ses collaborateurs [34]. Des agneaux nés de mères exposées au froid pendant la gestation, ont un $\mathrm{TAB}$ périrénal de poids identique, beaucoup plus riche en mitochondries et en UCP que celui des animaux témoins. Un mois plus tard, le tissu adipeux périrénal des agneaux issus du traitement expérimental est deux fois plus lourd et riche en lipides que celui des animaux témoins sans le maintien au même niveau de sa capacité thermogénique. Ces résultats tendraient à indiquer que, par le jeu de la conversion post-natale, l'importance du TAB à la naissance pourrait conditionner le développement ultérieur de la masse adipeuse totale.

Réinduire le TAB lorsque c'est possible et contrôler la thermogenèse réglable pourraient être envisagés comme un moyen efficace d'éliminer des calories excédentaires. L'expérimentation sur le chien citée précédemment, dans laquelle la réapparition du TAB par un traitement $\beta 3$-adrénergique est associée à une prise de poids plus faible, est en faveur de cette hypothèse [14].

\section{Conclusions}

Pendant longtemps, le terme de tissu adipeux recouvrait essentiellement le tissu adipeux blanc, considéré comme une entité, simple "accordéon ", capable de se distendre ou de régresser au gré des besoins de l'individu. La mise au point d'anticorps ou de sondes moléculaires capables de détecter la protéine découplante, caractéristique de l'adipocyte brun, dans toutes les espèces a permis de reconsidérer l'importance du TAB et des adipocytes bruns dans le fonctionnement de l'organisme en général. Le développement des cultures cellulaires a rendu possible la caractérisation des marqueurs de la différenciation adipocytaire utilisables in vivo. Les connaissances acquises par ces intermédiaires rendent mieux compte de la complexité des dépôts adipeux, de leur développement normal ou pathologique et de l'importance de l'innervation sympathique dans ces processus. L'ensemble des résultats souligne l'hétérogénéité métabolique et cellulaire des différents dépôts adipeux et permet de mettre en évidence une propriété importante des tissus adipeux : leur plasticité

\section{Summary}

Heterogeneity and cellular plasticity of adipose tissue

Two types of adipose tissues are usually considered : white and brown adipose tissues. The first one corresponds to the greatest energy buffer of the body and the second one dissipates energy as heat. These tissues play a crucial role in energy balance and their importance is different according to species. The relationship between white and brown adipocytes needs to be clarified for understanding the development of the whole adipose mass. Apparent conversion between these two tissues occurs during development and physiological or pathological situations. Recent results demonstrate the importance of the sympathetic nervous system on the development of adipose tissues as well as tissular or cellular plasticity.

\section{TIRÉS A PART}

L. Casteilla. 